\title{
Absolute Measurement of Electron Cloud Density
}

M. Kireeff Covo, A. W. Molvik, R. H. Cohen, A.

Friedman, P. A. Seidl, G. Logan, F. Bieniosek, D. Baca, J-L. Vay, E. Orlando, J. L. Vujic

June 22, 2007

Particle Accelerator Conference 2007

Albuquerque, NM, United States

June 24, 2007 through June 29, 2007 
This document was prepared as an account of work sponsored by an agency of the United States Government. Neither the United States Government nor the University of California nor any of their employees, makes any warranty, express or implied, or assumes any legal liability or responsibility for the accuracy, completeness, or usefulness of any information, apparatus, product, or process disclosed, or represents that its use would not infringe privately owned rights. Reference herein to any specific commercial product, process, or service by trade name, trademark, manufacturer, or otherwise, does not necessarily constitute or imply its endorsement, recommendation, or favoring by the United States Government or the University of California. The views and opinions of authors expressed herein do not necessarily state or reflect those of the United States Government or the University of California, and shall not be used for advertising or product endorsement purposes. 


\title{
ABSOLUTE MEASUREMENT OF ELECTRON CLOUD DENSITY*
}

\author{
Michel Kireeff Covo", Lawrence Livermore National Laboratory, Heavy-Ion Fusion Science Virtual \\ National Laboratory, Livermore, California 94550, USA and University of California at Berkeley, \\ 4155 Etcheverry Hall, MC 1730, Berkeley, California 94720, USA
}

Arthur W. Molvik, Ronald H. Cohen, and Alex Friedman, Lawrence Livermore National

Laboratory, Heavy-Ion Fusion Science Virtual National Laboratory, Livermore, California 94550, USA

\author{
Peter A. Seidl, Grant Logan, Frank Bieniosek, David Baca and Jean-Luc Vay, Ernest Orlando \\ Lawrence Berkeley National Laboratory, Heavy-Ion Fusion Science Virtual National Laboratory, 1 \\ Cyclotron Road, Berkeley, California 94720, USA
}
Jasmina L. Vujic, University of California at Berkeley, 4155 Etcheverry Hall, MC 1730, Berkeley, California 94720, USA

\begin{abstract}
Beam interaction with background gas and walls produces ubiquitous clouds of stray electrons that frequently limit the performance of particle accelerator and storage rings. Counterintuitively we obtained the electron cloud accumulation by measuring the expelled ions that are originated from the beam-background gas interaction, rather than by measuring electrons that reach the walls. The kinetic ion energy measured with a retarding field analyzer (RFA) maps the depressed beam space-charge potential and provides the dynamic electron cloud density. Clearing electrode current measurements give the static electron cloud background that complements and corroborates with the RFA measurements, providing an absolute measurement of electron cloud density during a $5 \mu$ s duration beam pulse in a drift region of the magnetic transport section of the High-Current Experiment (HCX) at LBNL.
\end{abstract}

\section{INTRODUCTION}

Particle and synchrotron radiation interaction with the background gas and walls can produce electrons. In some circumstances the created electrons can multiply and accumulate, deteriorating the beam quality and coupling with the beam to drive instabilities. This ubiquitous outcome is known as electron cloud and can generate electron-stimulated gas desorption, cloud-induced noise on instrumentation, tune shifts, instabilities and heat deposition on cryocooled components [1].

Electron Cloud Effects (ECE) were observed in the proton storage rings at BINP [2], the intersecting storage rings at CERN [3], the proton storage ring at LANL [4], the relativistic heavy ion collider at BNL [5], the photon factory at KEK [6,7], the low energy ring at KEKB [8], and other storage rings. They can potentially limit the performance of the large hadron collider (LHC) at CERN,

\footnotetext{
* This work was performed under the auspices of the U.S Department of Energy by University of California, Lawrence Livermore and

Lawrence Berkeley National Laboratories under contracts No. W-7405-

Eng-48 and DE-AC02-05CH11231.

"kireeffcovo1@1lnl.gov
}

and have been subject of and featured in various meetings.

Quantitative measurements of electron cloud density, in combination with simulation and theory, are fundamental for understanding ECE. Simple biased electrodes change the collection length and increase the energy of electron striking the electrodes. Previous measurements were made only between bunches by sweeping them towards a detector with a pulsed electrode $[4,9]$ or by selecting high energy electrons when the bunch pattern emerges [10].
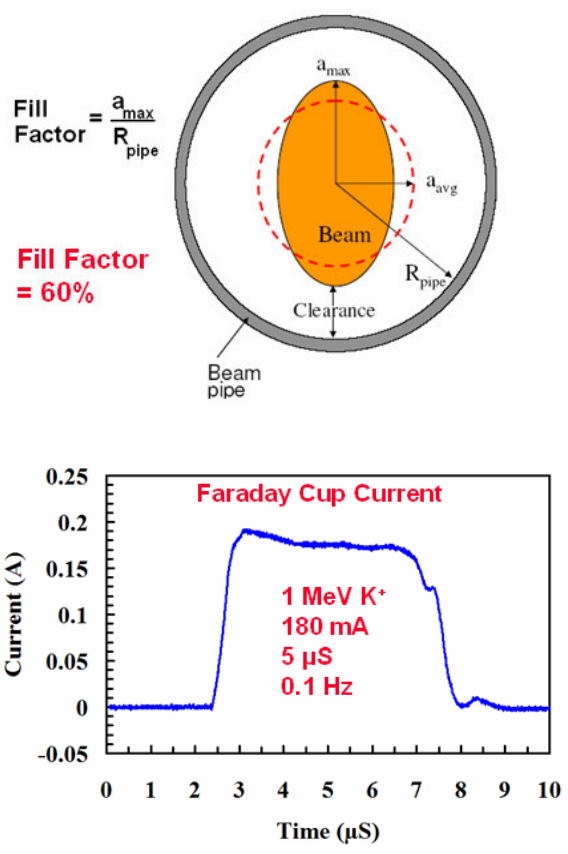

(a)

Figure 1: (a) Sketch of the transverse distribution of the beam, showing the $60 \%$ fill factor. (b) Faraday cup current of the beam, showing 0.18 A during $5 \mu$ s.

The High-Current Experiment (HCX) facility at LBNL is a driver scale single beam injector with a matching 
section, an electrostatic and magnetic transport section. It provides a $1 \mathrm{MeV} \mathrm{K}+$ ion beam every 10 seconds, consequently it does not have synchrotron radiation and electron multipacting; but it transports a beam current of 0.18 A for $5 \mu$ s with a $60 \%$ fill factor (Fig.1), therefore it has large interaction with the background gas and walls.

Inside the matching section and electrostatic quadrupole section the electrons are swept out by the electric field towards the positive rods, but inside the magnetic section the electrons can accumulate.

We developed two techniques, designated the "RFA technique" and "Clearing Electrode technique", which provide absolute measurement of time-dependent electron cloud density accumulation during the beam pulse [11].

\section{EXPERIMENTAL SETUP}

Fig. 2 shows the magnetic transport section from HCX that is at the background pressure of $\sim 5 \times 10^{-7}$ Torr. It has four room-temperature pulsed quadrupole magnets (QM1, QM2, QM3, and QM4) and three clearing electrodes (A, $\mathrm{B}$, and $\mathrm{C}$ ) in the drift regions.

Electrons can be confined radially by the beam spacecharge potential of $\sim 2 \mathrm{kV}$ and axially by the suppressor electrode (S) at one end and by the last electrostatic quadrupole at the other end, which are biased to $-10 \mathrm{kV}$ and $-18.6 \mathrm{kV}$, respectively.

Clearing electrodes $\mathrm{A}, \mathrm{B}$ and $\mathrm{C}$ can be biased to $+9 \mathrm{KV}$ in order to remove local sources of electrons when they drift to the gaps.

Electrons originating from ion impact on structures at the end of HCX can move upstream if the suppressor and clearing electrodes are turned off.

A Retarding Field Analyzer (RFA) [12] can replace the clearing electrode A. It is an extension of the RosenbergHarkay design [13] and has a linear motion feedthrough that allows positioning the RFA at $4 \mathrm{~cm}$ from the beam axis, where the beam space-charge equipotential (obtained using a three-dimensional self-consistent WARP code simulation) is near zero volt, matching the potential of grounded analyzer box and allowing measurements of ion and electron energy distribution.

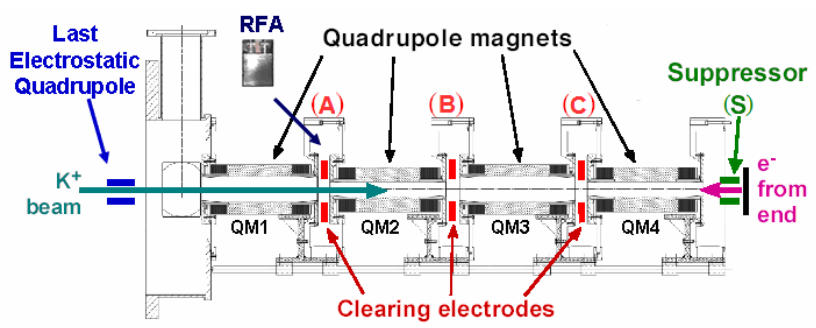

Figure 2: Magnetic quadrupole transport section of HCX. Electrons can be confined inside by the beam potential, if the suppressor and last electrostatic quadrupole are biased negatively. Local sources of electrons can be removed, if the clearing electrodes are turned on. RFA measures ions expelled by the beam potential, when the clearing electrode $\mathrm{A}$ is taken away.

\section{TECHNIQUES}

Electrons trapped during the beam passage are expelled at the end, when the beam potential decays. Estimation of electron cloud density from measurements of electron flux to the walls is not precise, because, at the same time that the electrons are expelled, the beam tail scrapes the wall desorbing new generated electrons that are indiscernible and confound measurements.

The electron accumulation was obtained by measuring the depressed beam potential. The beam interaction with the background gas produces ions and electrons. These charged particles are born inside a potential well that traps the electrons and expels the ions. The expelled ions convert their potential energy into kinetic energy when they reach the walls or enter the RFA with time-of-flight of a few hundred nanoseconds.

The maximum kinetic energy of the ions entering the RFA corresponds to the highest potential energy of the ions, i.e., the beam potential. As electrons accumulate, the beam potential decreases and so does the energy of the expelled ions [11,14].

The electron density as a function of time is obtained from the beam potential decay measurement accounting for the ion and electron transverse distributions.

WARP simulations show that the electron horizontal line charge density (e-_x) and vertical line charge density (e-_y) in Gap $\mathrm{C}$ at different times $(2-5 \mu \mathrm{s})$ have round Gaussian distribution (Fig. 3). The standard deviation $\sigma$ of $1.6 \mathrm{~cm}$ is obtained from the full width at half the maximum ( 2.335 times the standard deviation).

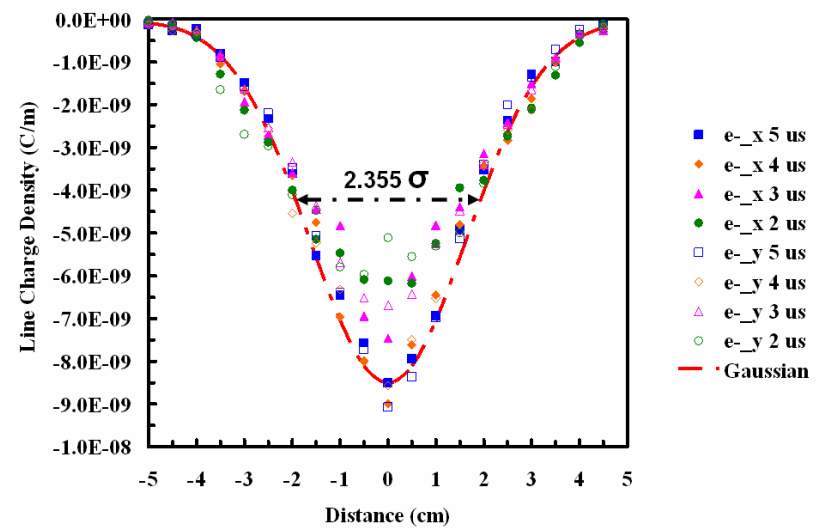

Figure 3: Vertical (e-_y) and horizontal (e-_x) line charge density of electrons in gap $\mathrm{C}$ at different times $(2 \mathrm{us}<\mathrm{t}<5$ us) obtained using a three-dimensional self-consistent particle in cell WARP code.

The electron potential for a round Gaussian transverse electron distribution is:

$U_{\text {electron }}(R)=-\frac{\lambda}{4 \pi \varepsilon_{0}} \int_{0}^{R} \frac{2}{t}\left[1-e^{-\frac{t^{2}}{2 \sigma^{2}}}\right] d t$

where $\lambda$ is the electron line density, $\sigma$ is the standard deviation, and $\mathrm{R}$ is the beam pipe radius. 
If we substitute $\sigma=1.6 \mathrm{~cm}$ and $\mathrm{R}=4 \mathrm{~cm}$ and integrate it numerically, we obtain:

$U_{\text {electron }}(R) \cong-\frac{\lambda}{4 \pi \varepsilon_{0}} * 1.79$

In the same way, the simulations show that the distribution is uniform for the ions and has a fill factor of $\sim 60 \%$.

The electric field for a uniform beam with $60 \%$ fill factor can be derived from the Gauss law and integrated for $0<\mathrm{r}<\mathrm{R}$, giving the beam potential of:

$$
U_{\text {beam }}(R) \approx \frac{\lambda}{4 \pi \varepsilon_{0}} * 3.01
$$

Consequently the electron line density need to be 1.68 times the beam line density in order to induce the same space-charge potential strength contribution.

The RFA technique obtains the dynamic electron cloud density by measuring the reduction of the beam potential and multiplying it by 1.68 to take into account the different transverse distributions. As the ions need several hundreds nanoseconds to be expelled, the RFA technique cannot measure a constant background electron density created at the beginning of the beam passage, when the beam potential increases.

The density measured with the RFA can be supplemented and corroborated by clearing electrodes measurements, giving the absolute electron density.

The clearing electrode $\mathrm{A}$, which is at the same axial position as the RFA, measures the current of electrons drifting to gap A. The electron line charge is obtained dividing the electron current by the average drift velocity $\left(\overline{v_{d}}\right)$ of the electrons inside the quadrupoles.

Clearing electrode measurements show that, when the suppressor and clearing electrodes are off, electrons produced at the end wall structures drift through each of the quadrupole magnets at a velocity of $0.60 \pm 0.11 \mathrm{~m} / \mu \mathrm{s}$. This value was obtained by dividing the time that the electrons need to reach the successive clearing electrodes by the effective magnetic field length of the quadrupole $(\sim 32 \mathrm{~cm})$ [15] and it is in excellent agreement with the value of $0.66 \mathrm{~m} / \mu \mathrm{s}$ [16] predicted by the simulations.

The average drift velocity has $\vec{E} \times \vec{B}$ and $\overrightarrow{\nabla B}$ contributions components. The first component is directly proportional to the electric field. The second component is a function of the kinetic energy of the wall-desorbed electrons, which is proportional to the beam potential and consequently it is proportional to the electric field. Therefore $\overline{v_{d}}$ is assumed directly proportional to the electric field, which decreases with beam neutralization.

The constant background electron density created at the beginning of the beam passage is determined by measuring the clearing electrode $\mathrm{A}$ current with the clearing electrodes B, C and the suppressor S biased and by dividing it by $\overline{v_{d}}$.

\section{BEAM DENSITY MEASUREMENTS}

Figs. 4 and 5 show an experiment that was performed intentionally switching off the clearing electrodes B and C between the magnets and the suppressor $\mathrm{S}$ at the end of the magnetic transport section, in order to increase sources of electrons and measure the different electron accumulation during the beam passage.

Fig. 4 shows the Faraday cup current (blue line), corrected for the time of flight to the RFA axial location to indicate the timing of the process, using the left-hand ordinate axis. It also shows the dynamic beam potential decaying with time, using the right-hand ordinate axis, for three different conditions. For the first condition the clearing electrodes and the suppressor are all on. The depressed beam potential (red squares) has a similar slope to the Faraday cup current, implying that there is no significant beam neutralization during the beam pulse. For the second condition the clearing electrodes are off and the suppressor is on, which allows electrons from local sources to accumulate, depressing the beam potential by $12 \%$ (pink triangles). For the third condition the suppressor and the clearing electrodes are off, which also allows electrons to drift upstream, depressing the beam potential by $43 \%$ (green circles).

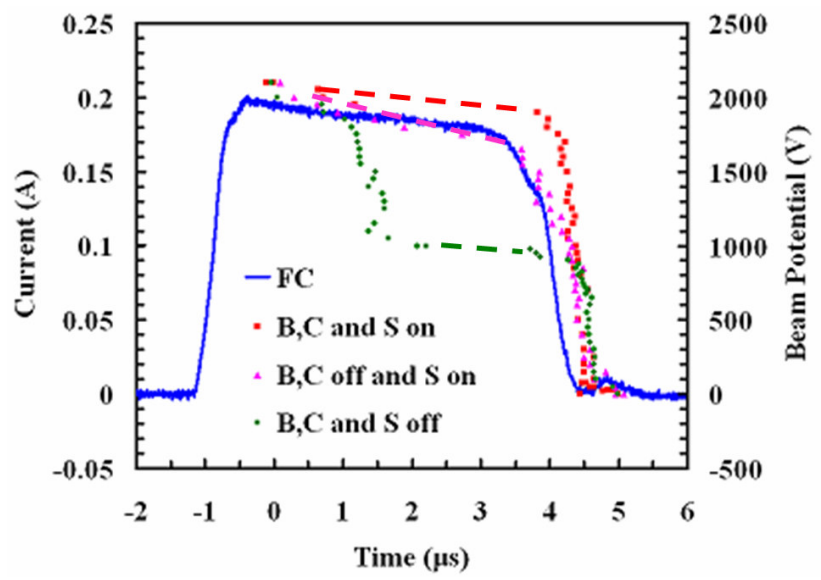

Figure 4: Faraday cup current and dynamic beam potential measured for three different configurations, increasing the sources of electrons. For the first condition (B, C, and $\mathrm{S}$ on), the clearing electrodes $\mathrm{B}, \mathrm{C}$ and suppressor $\mathrm{S}$ are on. For the second condition (B, C, off and $\mathrm{S}$ on), we allow local sources of electrons to accumulate by turning off the clearing electrodes $\mathrm{B}$ and $\mathrm{C}$. For the third condition (B, C, and S off) we also allow electrons generated at the end structures to drift upstream by turning off the suppressor S. The dashed red, pink and green lines are placed for better visualization.

In Fig. 5, the red and green lines denote the electron currents measured with clearing electrode A, after subtracting the beam induced capacitive signal, when clearing electrodes B, C and the suppressor S are on and off, respectively. The pink line is the sum of the currents from clearing electrodes $\mathrm{A}, \mathrm{B}$ and $\mathrm{C}$ minus the beam induced capacitive signal, when clearing electrodes A, B, 
$\mathrm{C}$ and the suppressor $\mathrm{S}$ are on. It corresponds to the electron current that accumulates inside the magnetic section when the clearing electrodes are off and the suppressor is on.

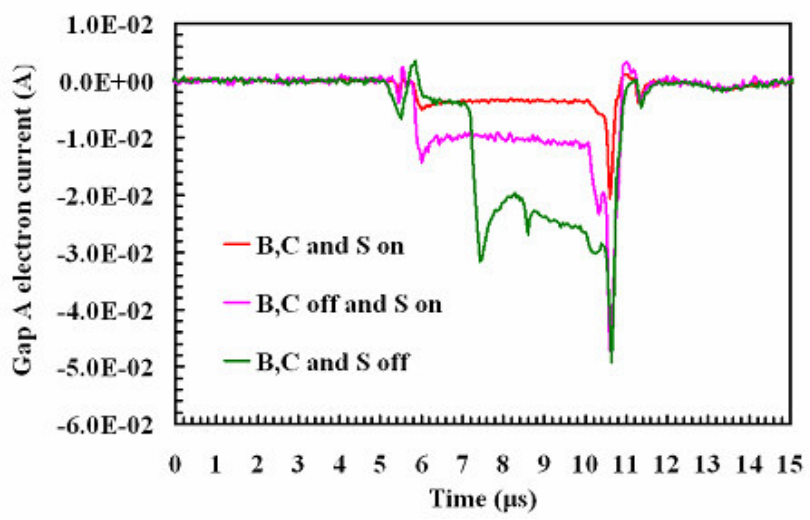

Figure 5: Electron current from clearing electrode A obtained for the same configurations of Fig. 4, after subtracting the beam induced capacitive signal. As $\overline{v_{d}} \sim 0.60 \mathrm{~m} / \mu \mathrm{s}$ for the first configuration (clearing electrodes B, C and suppressor S on) is known, we can use the depressed beam potential measured with the RFA to obtain $\overline{v_{d}}$ for the other configurations.

The beam neutralization, defined as the ratio of electron line charge density divided by ion line charge density, obtained for the clearing electrode and RFA techniques are in good agreement and are summarized in the table 1.

Table 1: Comparison of the beam neutralization measured in gap A using the clearing electrode and RFA techniques.

\begin{tabular}{|l|l|l|l|}
\hline $\begin{array}{l}\text { Beam } \\
\text { neutralization }\end{array}$ & $\begin{array}{l}\text { B, C and } \\
\text { S on }\end{array}$ & $\begin{array}{l}\text { B, C off } \\
\text { and S on }\end{array}$ & $\begin{array}{l}\text { B, C and } \\
\text { S off }\end{array}$ \\
\hline Clear. Electrodes & $7.3 \%$ & $25.2 \%$ & $89.2 \%$ \\
\hline RFA & $7.3 \%$ & $27.5 \%$ & $79.5 \%$ \\
\hline
\end{tabular}

When the clearing electrodes are initially on and they are turned off sequentially $(\mathrm{C}, \mathrm{B}$ and $\mathrm{A})$, the current measured in the last active clearing electrode is the same, meaning that the beam neutralization of gaps A, B and C, Fig. 2, will be the same after a while if the clearing electrodes and suppressor are off. The RFA technique is in excellent agreement with the beam neutralization of $80.7 \%$ in gap C obtained with simulation.

Another alternative analysis of clearing electrode data is obtained from Fig. 5. As we said before, when the clearing electrodes $\mathrm{B}, \mathrm{C}$ are off and the suppressor $\mathrm{S}$ is on, the whole magnetic transport section will work as an electron trap. If we integrate the pink line over the beam duration and divide it by the distance between last electrostatic quadrupole and the suppressor, it gives a beam neutralization of $27.2 \%$ and is in excellent agreement with the Table 1 results.

\section{CONCLUSIONS}

Rather than estimating electron cloud density by the flux of electrons to the walls, we estimate the dynamic density by probing the depressed beam potential, measuring the energy of the expelled ions from the interaction of the beam with the background gas, and correcting it for the different electron and ion transverse distribution. The dynamic density is complemented and corroborated by measurements from the Clearing Electrode technique, giving an absolute density.

Sources of electrons are intentionally added in the magnetic transport section of the HCX by turning off the clearing electrodes B and C, and suppressor S. Table 1 summarizes the results obtained with the RFA and Clearing Electrode techniques, which are in reasonable agreement. As a future work, we are planning to use an electron gun to inject a known amount of electrons to crosscheck the neutralization obtained with our new techniques. We are also developing a new diagnostic that is weakly capacitively coupled with the beam and can obtain the beam neutralization in a single shot.

\section{ACKNOWLEDGMENTS}

We wish to thank Tak Katayanagi who built the RFA, Wayne G. Greenway who maintains HCX, and Craig Rogers and William L. Waldron who provided electronic support. We also want to express our gratitude to Richard A. Rosenberg and Katherine C. Harkay for sharing details that aided our RFA design, and to Miguel Furman for his insightful comments. This work was performed under the auspices of U.S. Department of Energy by the University of California, LLNL and LBNL under contracts No. W7405-ENG-48, and No. DE-AC02-05CH11231.

\section{REFERENCES}

[1] H. Fukuma, in: Proc. of Diag. Instrum. Part. Acc. Conf., Lyon, France, 2005, p. 122.

[2] G. Budker, G. Dimov, and V. Dudnikov, Sov. Atom. E. 22, 5 (1967).

[3] O. Gröbner, in: Proc. of 10th Intern. Acc. Conf., Serpukhov, 1977, p. 277.

[4] R. J. Macek, A. Browman, D Fitzgerald, R. McCrady, F. Merrill, M. Plum, T. Spickermann, T. S. Wang, J. Griffin, K.Y. Ng, D. Wildman, K. Harkay, R. Kustom, and R. Rosenberg, in: Proc. of Part. Acc. Conf., Chicago, IL, 2001, p. 688.

[5] S. Y. Zhang, W. Fischer, H. Huang and T. Roser, in: Proc. of Eur. Part. Acc. Conf., Lucerne, 2004, p. 944.

[6] M. Izawa, Y. Sato, and T. Toyomasu, Phys. Rev. Lett. 74, 5044 (1995).

[7] K. Ohmi, Phys. Rev. Letters 75, 1526 (1995).

[8] J. W. Flanagan, K. Ohmi, H. Fukuma, S. Hiramatsu, M. Tobiyama, E. Perevedentsev, Phys. Rev. Lett. 94, 054801 (2005).

[9] R. J. Macek, A. Browman, M. Borden, D. Fitzgerald, R. McCrady, T. Spickermann, and T. Zaugg, in: Proc. of Part. Acc. Conf., Portland, OR, 2003, p. 3089. 
[10] K. Kanazawa, H. Hukuma, H. Hisamatsu, and Y. Suetsugu, in: Proc. of Part. Acc. Conf., Knoxville, TN, 2005, p. 1054.

[11] M. Kireeff Covo, A. W. Molvik, A. Friedman, J.-L. Vay, P. A. Seidl, G. Logan, D. Baca, and J. L. Vujic, Phys. Rev. Lett. 97, 54801 (2006).

[12] M. Kireeff Covo, A. W. Molvik, A. Friedman, J. J Barnard, P. A. Seidl, G. Logan, D. Baca, and J. L. Vujic, Nucl. Instr. and Meth. A 577, 139 (2007).

[13] R.A. Rosenberg, K. Harkay, Nucl. Instr. and Meth. A 453 (2000) 507.
[14] J. Klabunde, H. Reiserb, A. Schijnlein, P. Spadtke, and J. Struckmeier, in: Proc. of Part. Acc. Conf., Santa Fe, NM, 1983, p. 2543.

[15] P. A. Seidl , M. Kireeff Covo, D. Baca, A. Faltens, A. W. Molvik, G. Ritchie, G. Sabbi, and D. Shuman, Nucl. Instr. and Meth. A 544, 486 (2005).

[16] R. H. Cohen, A. Friedman, S. M. Lund, A. W. Molvik, E. P. Lee, T. Azevedo, J.-L.Vay, P. Stoltz, and S. Veitzer, Phys. Rev. ST AB 7, 124201 (2004). 